\title{
Treinamento de um aluno em Algoritmos e Ciência de Dados, como conhecimento adicional a sua grade acadêmica de Engenharia Química
}

DOI: 10.37702/2175-957X.COBENGE.2021.3737

Reginaldo Arakaki - reginaldo.arakaki@poli.usp.br

Universidade de São Paulo

RUA SOLDADO JOAO DE OLIVEIRA 369

05140-000 - SÃO PAULO - SP

Tiago Yukio Fujii - tiago.fuji@usp.br

Universidade de São Paulo

Rua Gesselina Ré Pinto 68

13338-245 - Indaiatuba - SP

Leonardo Schirrmeister Marchetti - leonardomarchetti@estudante.ufscar.br Universidade Federal de São Carlos rua Antonio de Gouveia Giudice 1011

05460-001 - São Paulo - SP

Resumo: Este artigo descreve resultados obtidos por um estudante de Engenharia Química em uma iniciativa de treinamento de 12 meses em programação computacional. O objetivo do estudante foi aprender e aprimorar projeto de algoritmos, métodos de implementação, ciclo de correção de erros, iniciação em técnicas de inteligência artificial e machine learning. O plano foi baseado na taxonomia de Bloom, para evidenciar aspectos pedagógicos e medir os avanços de aprendizado dos desafios através do resultados obtidos com o estudante. Habilidades adicionais de soft skills importantes para a profissão como autonomia, resiliência para enfrentar erros e dificuldades foram evidenciadas e documentadas

Palavras-chave: Educação remota, Avaliação pedagógica, Ciência de Dados, Métodos de Programação, Machine Learning, Ferramentas de Processamento de Dados, Soft-skills 


\section{Treinamento de um aluno em Algoritmos e Ciência de Dados, como conhecimento adicional a sua grade acadêmica de Engenharia Química}

\section{INTRODUÇÃO}

Nenhuma profissão, seja de humanas ou de exatas, pode dispensar o uso da computação, de plataformas digitais ou de uma integração digital. Os exemplos são inúmeros e um que ilustra bem hoje são os profissionais de direito aprendendo técnicas de programação para construir um contrato digital em plataformas baseadas em blockchain ${ }^{1}$ (FGV, 2021).

Nesse contexto, adicionalmente a uma disciplina de Algoritmos e Programação Computacional, da grade curricular da área de Engenharia Química (UFSCAR, 2021), (USP-LORENA, 2021), foi estabelecida e criada uma iniciativa de estudos de técnicas computacionais visando ciência de dados e inteligência artificial, em nível de iniciação científica. Os resultados foram positivos e permitiu o entendimento dos níveis de conhecimento inicial e os resultados finais obtidos ao final de 12 meses de atividades.

A iniciativa constou de uma meta de aprendizado pedagógico nas seguintes técnicas de computação: a) Como projetar algoritmos? b) Adoção de ferramentas para exercícios práticos para tornar tangível os aspectos abstratos de algoritmos; c) Entendimento de técnicas de sistemas baseados em internet com criação de serviços interativos com base de dados; d) Estudos de técnicas de inteligência artificial, com foco em aprendizado de máquinas; e) Publicar um artigo com relatos das atividades realizadas e resultados obtidos. O projeto pedagógico desta iniciativa envolveu notação e pensamento algorítmico; linguagem de programação Python, Ferramenta de tratamento de dados; e iniciação em Inteligência Artificial e Machine Learning.

É sempre importante destacar o potencial do conhecimento em computação citado: na graduação (em atividades de IC), na indústria em estágio e também como profissional e na pós-graduação em pesquisa. Durante a graduação, o potencial de se construir projetos de processos químicos com requisitos de automação e inteligência computacional pode fazer a diferença. E é muito provável que isso aconteça durante a carreira profissional.

O objetivo deste artigo é o de descrever a iniciativa de formação adicional em computação para um aluno da área de engenharia química, no nível de iniciação científica. Inclui o projeto didático, atividades realizadas e resultados obtidos.

\section{O PROJETO DE INICIAÇÃO EM CIÊNCIA DE DADOS E ALGORITMOS}

O grande propósito em que os professores e o estudante se engajaram foi o de estabelecer um caminho promissor de conhecimentos que permitisse que o nível iniciante em computação do estudante - apenas usuário de dispositivos eletrônicos - pudesse alcançar um conhecimento em computação nos aspectos de programação, projeto de sistemas e adoção de técnicas de inteligência artificial e aprendizado de máquina (machine learning).

\footnotetext{
${ }^{1}$ Blockchain Plataforma que implementa um poderoso protocolo de segurança em termos de autenticidade e não repúdio. É uma plataforma base de moedas digitais como o bitcoin.
} 
O desdobramento do desafio foi a de montagem de um plano sustentado de aprendizado conceitual e prático em termos de métodos de programação, organização de um sistema de software, plataformas internet e inserção de mecanismos de inteligência artificial. Para introduzir e familiarizar-se com os conceitos necessários sobre aprendizado de máquina e Deep Learning, foram recomendados cursos (COURSERA, 2021) e apresentações (AMINI, 2021) disponíveis gratuitamente. Os requisitos prévios esperados do aluno são conhecimentos básicos na linguagem de programação Python, devido ao grande acervo de bibliotecas voltadas à ciência de dados, além de fundamentos sobre álgebra linear e cálculo integral e diferencial, necessários para compreender a notação utilizada na formulação teórica do aprendizado das redes neurais.

A partir destes materiais, foi possível introduzir o aluno sobre o treinamento de redes neurais com foco principal em redes neurais profundas, sua fundamentação teórica, e como aplicá-las em sua área de conhecimento. As redes neurais profundas podem ser utilizadas para modelar relações não lineares complexas, auxiliando na tomada de decisão e automação de processos envolvendo visão computacional, processamento de linguagem natural, e reconhecimento de voz. Além disso, foi possível familiarizar-se com as principais bibliotecas utilizadas na área de aprendizado de máquina e ciência de dados, como Pandas e Numpy para processamento de dados, Matplotlib para visualização, e TensorFlow e Keras para modelagem de redes neurais.

\section{A METODOLOGIA APLICADA}

A metodologia aplicada nesta iniciativa foi baseada nos seguintes requisitos: a) Pouco conhecimento em programação; b) Ferramentas de implementação práticas boa aceitação acadêmica e industrial; c) Método e técnicas para projeto e construção de sistemas digitais; d) $\mathrm{O}$ uso de dados como elemento diferenciado para obtenção dos resultados. Com isso, organizou-se a seguinte metodologia para sustentar este projeto de formação (Figura 3.1).

Figura 3.1 - Metodologia aplicada no Projeto.

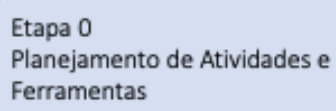

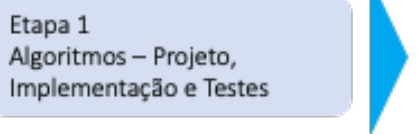

Etapa 2

Ferramentas de Tratamento de Dados e Internet

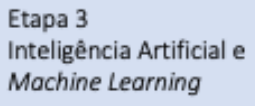

Para atender ao requisito de nível de conhecimento a avaliação por entrevistas sobre fundamentos de lógica de programação definiu conhecimento básico necessitando a assimilação de métodos de construção de lógica algorítmica, com base em componentes de programação estruturada baseada em notação gráfica de Nassi-Shneiderman (Arakaki, 1990), (Pressman, 2016). Nesta atividade de lógica de programação usando técnicas estruturadas, organizou-se o plano de aprendizado conforme indicado na Figura 3.2. Importante observar que a interação com o aluno para estabelecer o conhecimento básico e identificar o potencial de evolução permitiu o planejamento. Considerando a taxonomia de Bloom (VALENTE, 2013) como referência, as metas consideradas de aprendizado foram 4, listadas a seguir: 
1. Técnica: Lógica algorítmica estruturada

- Meta Estabelecida: Lembrar e entender estruturas lógicas de programação;

- Habilidades adicionais estimuladas: Autonomia na busca de conhecimentos através de meios especializados como revistas técnicas e aprendizado prático com ciclos de detecção de erros e correção;

- Método aplicado: Conceitos, exercícios e acompanhamentos práticos;

2. Técnica: Implementação Algorítmica e Iniciação em Ciência de Dados

- Meta Estabelecida: Entender e criar estruturas lógicas de programação. Manipulação de grandes conjuntos de dados;

- Habilidades adicionais estimuladas: Resiliência na construção prática, baseada em métodos cíclicos da criação: executar, detectar erros e ajustar e repetir. Entender os dados como elemento importante de argumentação e evidências no mundo da transformação digital em curso;

- Método aplicado: Implementar na prática os exercícios conceituais utilizando a linguagem Python com acompanhamentos práticos;

3. Técnica: Serviços Web

- Meta Estabelecida: Entender e criar estruturas lógicas para publicar um site na internet: páginas e serviços;

- Habilidades adicionais estimuladas: Adoção de ferramentas adicionais às de seu conhecimento, através da atitude de integração: ou seja, cada ferramenta tem a sua utilidade, mas podem se combinar para compor sistemas mais complexos. A empatia em relação aos usuários de sistemas para adicionar boas experiências de uso.

- Método aplicado: Implementar na prática os exercícios conceituais utilizando a linguagem Python com a acompanhamentos práticos, integrar com bibliotecas de publicação de páginas web (Flask) e plataformas de persistência de informações (MongoDB);

4. Técnica: Iniciação em Inteligência Artificial e Machine Learning

- Meta Estabelecida: Entender os fundamentos de técnicas de aprendizado de máquina. Aplicar as técnicas em um domínio de problema prático;

- Habilidades adicionais estimuladas: Análise crítica de plataformas digitais baseados em inteligência artificial pode trazer novas possibilidades para a Engenharia. Entender a possibilidade de criar inovações de processos e métodos baseando-se nas práticas digitais;

- Método aplicado: Juntar o conhecimento de linguagem de programação, ferramentas de manipulação de grandes massas de dados (Pandas, Numpy e MongoDB), estudar, configurar e testar modelos de Machine Learning (TensorFlow e Keras) aplicados para o reconhecimento de imagens.

Figura 3.2 - Planejamento para adicionar conhecimento computacional.

\begin{tabular}{|l|l|l|l|l|}
\hline & 10 Trim & 20 Trim & 3 Trim & 40 Trim \\
\hline Lógica de Programação & & & & \\
\hline Implementação Algorítmica & & & & \\
\hline Serviços Web & & & & \\
\hline Iniciação em IA e ML & & & & \\
\hline
\end{tabular}


As etapas 1, 2 e 3 foram executadas conforme indicados no item 4 e 5 do artigo.

No item 5 , as metas pedagógicas foram avaliadas.

\section{ATIVIDADES PRÁTICAS E APRENDIZADOS}

Cada atividade planejada foi desenvolvida segundo o planejamento e alguns resultados técnicos e não técnicos são comentados e avaliados.

\subsection{Lógica de Programação}

As estruturas de programação usando diagramas NS (Nassi-Scheneirdman) compreendendo sequência, decisão (If-Then-Else), loops (For e While) e Múltiplos-Blocos foram exercitados trabalhando-se em lógicas de listas lineares, ordenação e formatação de impressão.

Adicionalmente, o contexto da transformação digital, o perfil dos profissionais graduados de diversas áreas e a relação com plataformas digitais foram objetos de compartilhamento entre alunos e professores. Dentre essas discussões, a Inteligência Artificial (I.A.) foi um tema presente quando se analisa o avanço da computação e da nossa sociedade atual, há uma grande quantidade de dados sendo gerados diariamente com a ampliação do uso da internet por meio de aplicativos e serviços oferecidos por diversas empresas e, consequentemente, computadores geram milhares de informações para os seus bancos de dados diariamente. A Machine Learning e a I.A. (ZHUANG, 2020) foram tecnologias desenvolvidas e aperfeiçoadas para conseguir identificar padrões e interpretar os muitos dados obtidos.

Muitas instituições públicas e privadas do mercado utilizam essa tecnologia para se desenvolver e oferecer diversos produtos e serviços, atualmente é comum ver em carros autônomos, casas inteligentes, assistentes virtuais como Siri e Alexa, porém a I.A. é aplicada em outras diversas áreas, na medicina por exemplo, para análise de exames e diagnósticos, e nas indústrias, com o movimento da Indústria 4.0 (RIBEIRO, 2020) buscando otimização e aperfeiçoamento dos processos produtivos industriais.

Como resultado desta atividade, segundo o planejamento indicado no item 3 (Figura

Figura 4.1 - Resultados obtidos da Meta 1.

Meta Pedagógica: Lógica de Programação

\begin{tabular}{|l|l|l|l|l|}
\hline 1. Lembrar & 2. Entender & 3. Aplicar & 4. Avaliar & 5. Criar \\
\hline Atendido & Atendido & Atendido & Não & Não \\
\hline
\end{tabular}

Habilidades Adicionais (Softskills)

\begin{tabular}{|l|l|l|}
\hline Autonomia & Pesquisa & Ciclo erro \\
\hline $\begin{array}{l}\text { Básica em enfrentar desafios de } \\
\text { exercícios de lógica. }\end{array}$ & Iniciado em sites, artigos e livros. & $\begin{array}{l}\text { Entendido e praticado no papel. Ainda difícil pela } \\
\text { abstração conceitual. }\end{array}$ \\
\hline
\end{tabular}

Nesta atividade, a adoção de uma ferramenta Python foi a tática adotada para estímulo baseado na prática. 


\subsection{Implementação Algorítmica e Iniciação em Ciência de Dados}

Com esse conhecimento desenvolvido após treinos repetitivos buscou-se iniciar a programação em Python para aplicações práticas. Dessa forma foi necessário entender o funcionamento do ambiente de desenvolvimento interativo Jupyter Notebook, criando e organizando os arquivos em linguagem Python, e como executar os códigos. Dominando o funcionamento do ambiente base para o estudo, voltou-se a atenção para a sintaxe da linguagem, compreendendo o funcionamento das strings, dos números int, float, long e complex, dos booleanos, de dicionários, de arrays, de matrizes, e métodos básicos para modificar seu conteúdo, como no caso das strings, as funções len(), lower(), upper() e entre outras.

Continuando com as bases da linguagem, focou-se nas estruturas de lógica e de repetição, como if/elif/else, for, while, def, class e with, utilizando projetos periódicos simples para treino e assimilação dos blocos e da endentação que o Python utiliza. Em conjunto, e já aprofundando, aplicou-se nesses projetos alguns módulos que acompanham a biblioteca do Python (numpy, pandas, random, datetime etc.), assim, foi necessário o conhecimento de importação de bibliotecas e as particularidades de cada uma para os projetos estabelecidos.

Finalizando a etapa inicial do desenvolvimento do conhecimento em Inteligência Artificial, aplicou-se os conceitos tratados em um projeto com arquivos em formato csv e xlsx. Utilizando a biblioteca csv, em conjunto com o matplotib e um arquivo OpenSource desenvolveu-se o entendimento da importação e edição deste arquivo, assim como a representação gráfica dos dados presentes, facilitando a visualização e a interpretação de uma base de dados grande agrupando informações com poucas linhas de código.

Persistir os dados foi o próximo passo, sendo desenvolvido um projeto utilizando o MongoDB com o intuito de aprofundar o aprendizado em base de dados e criação de sistemas para alimentação dessas bases. O projeto constituiu-se em um sistema de doação de remédios que não são mais utilizados, onde o usuário poderia realizar doações e/ou receber os remédios em um estoque disponível em pontos de coleta cadastrados, tanto os remédios quanto os pontos de coleta desenvolvidos eram fictícios, apenas com o intuito da compreensão dos conceitos de base de dados.

O MongoDB (MONGODB, 2021) é um gerenciador de banco de dados no formato JSON orientado a documentos, sendo este o mais utilizado no mercado e é possível armazenar esses dados sem se preocupar com a estrutura. Aplicando no projeto proposto, utilizou-se 6 classes para a base de dados (Doação, Estoque, Pontos de Coleta, Recebimento, Remédios e Usuário). Ver figura 4.2.

A classe Usuário é responsável por manter os dados dos usuários com suas informações pessoais (nome, data de nascimento, e-mail, cep etc.), e mantém os registros das movimentações dentro da plataforma, caso ele tenha realizado uma doação ou recebido algum remédio. A classe Remédios contém os remédios cadastrados, assim como a bula e fabricante, os Pontos de Coletas também mantêm o registro de todos os pontos de coleta cadastrados, com as informações do local (nome, endereço e CEP) e cada um possuindo seu estoque próprio. 
Figura 4.2 - Diagrama de interligação entre classes do projeto.

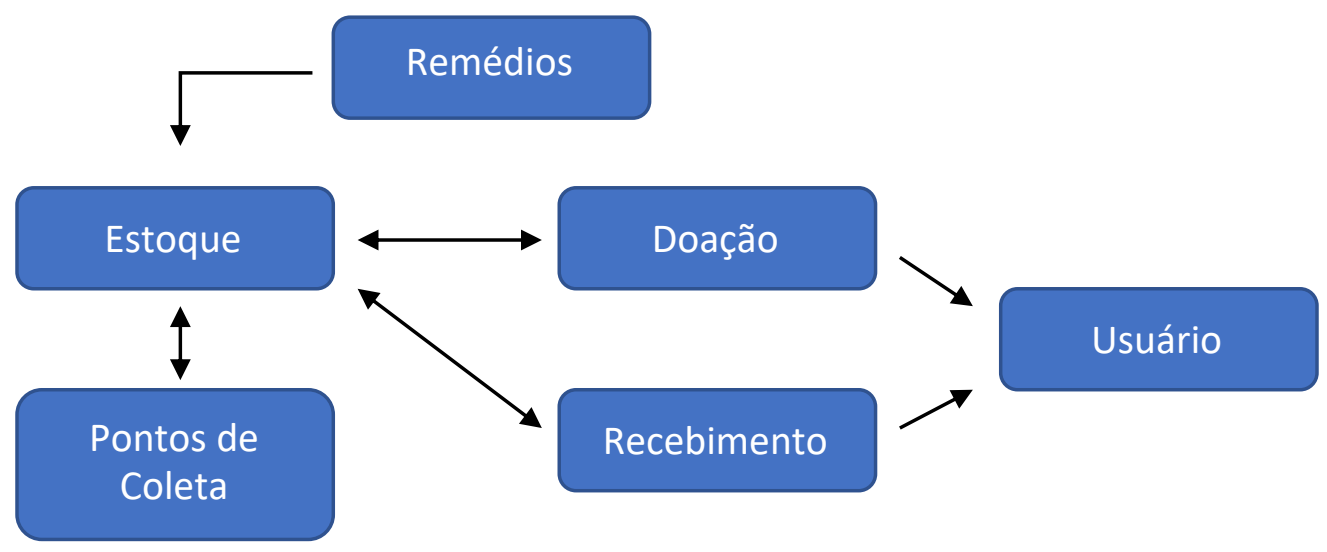

Como resultado desta atividade, segundo o planejamento indicado no item 3 (Figura 4.3).

Figura 4.3 - Resultados obtidos da Meta 2.

\begin{tabular}{|l|l|l|l|l|}
\hline Meta Pedagógica: Implementação Algorítmica \\
\hline 1. Lembrar & 2. Entender & 3. Aplicar & 4 . Avaliar & 5. Criar \\
\hline Atendido & Atendido & Atendido & Iniciado & Atendido \\
\hline Habilidades Adicionais (Softskills) & Dados como evidência & Ciclo erro \\
\hline Resiliência & $\begin{array}{l}\text { A ferramenta de manipulação de } \\
\text { grandes volumes de dados } \\
\text { executar um algoritmo no papel é partecter os erros e, num ciclo } \\
\text { interativo e metodológico, gerar a } \\
\text { programação correta. }\end{array}$ & $\begin{array}{l}\text { Entendido e praticado no computador. A } \\
\text { plataforma notebook Jupyter ajudou nos desafios } \\
\text { possibilidicos para lidar com os erros como importância de } \\
\text { instrumento de aprendizado. }\end{array}$ \\
\hline
\end{tabular}

Segundo o estudante, o seguinte destaque: "Ao final desta primeira etapa foi possível alcançar um maior conhecimento sobre as aplicações da computação no cotidiano, assim como a lógica de programação, como conseguir identificar padrões e repetições em desafios propostos, dessa forma contribuindo para a interpretação de problemas e organização das etapas necessárias para a resolução. Ao mesmo tempo, foi possível praticar esses conhecimentos nos projetos menores que realizamos e conhecer algumas bibliotecas importantes na linguagem Python. Especificamente, o conhecimento de estruturação de uma base de dados, e como as informações podem ser armazenadas, concomitantemente desenvolveu-se o entendimento da construção de um banco de dados com um projeto de maior complexidade aplicando constantemente os conhecimentos desenvolvidos da lógica de programação e as bibliotecas. Aproximando esta etapa de aplicações na área da Engenharia Química, foi possível visualizar aplicações de dados gerados em indústrias químicas." 


\subsection{Página WEB (Flask/MongoDB)}

Avançando no projeto da doação de remédios e tornando-o mais visual, desenvolveu-se uma página web utilizando a biblioteca Flask do Python (PYTHON SOFTWARE FOUNDATION, 2021). O Flask é um arcabouço modular que possibilita o desenvolvimento de aplicações web, dessa forma construiu-se uma página com host local vinculada à base de dados MongoDB criada anteriormente, a qual consulta os dados através do site.

Na página, é possível visualizar informações importantes do projeto, como o número de usuários, quais os pontos de coleta e os estoques de cada uma, a quantidade de cada remédio disponível e entre outras. Esta etapa do processo de aprendizado visa aplicar os conhecimentos das bibliotecas matplotib, numpy e pandas com a base de dados criada no projeto com MongoDB, e representar os dados de uma forma intuitiva e visualmente atrativa, assim como desenvolver o conhecimento básico sobre a construção de páginas web.

Ao final da criação da página web foi desenvolvido o raciocínio e a estruturação básica da criação de um site, vinculado ao programa criado anteriormente para apresentar os dados direto da base de dados, tornando a experiência de visualização das informações coletadas mais agradável. Esta etapa do processo não se estendeu, pois o intuito era criar o contato com a plataforma Flask e reconhecer as possibilidades e as dificuldades na criação de uma página web.

Como resultado desta atividade, segundo o planejamento indicado no item 3 (Figura 4.4).

Figura 4.4 - Resultados obtidos da Meta 2.

\begin{tabular}{|l|l|l|l|l|}
\hline Meta Pedagógica: Serviço web & 4. Aplicar & Avaliar & 5. Criar \\
\hline 1. Lembrar & 2. Entender & Atendido & Atendido & Iniciado \\
\hline Atendido & Atendido & Visão de integração \\
\hline Habilidades Adicionais (Softskills) & $\begin{array}{l}\text { Integração de ferramentas para solução: base de dados } \\
\text { (Mongo DB), servidor de páginas (Flask e Apache), servidor } \\
\text { de lógica (Flask e Python) e manuseio de dados em grandes } \\
\text { volumes (Pandas). }\end{array}$ \\
\hline $\begin{array}{l}\text { A diferença entre um programa feito para executar num } \\
\text { computador e construir um site acessível por muitos usuários, } \\
\text { através da internet, inclui tecnologia e, mais importante, } \\
\text { modelos de negócio. Foi iniciado a reflexão sobre. }\end{array}$
\end{tabular}

Segundo o estudante, o seguinte destaque: "Ao final da criação da página WEB foi desenvolvido o raciocínio e a estruturação básica da criação de um site, vinculado ao programa criado anteriormente para apresentar os dados direto da base de dados, tornando a experiência de visualização das informações coletadas mais agradável. Esta etapa do processo não se estendeu, pois o intuito era criar o contato com a plataforma Flask e reconhecer as possibilidades e as dificuldades na criação de uma página WEB." 


\subsection{Conceitos de IA e Machine Learning}

O estudo de redes neurais e IA utilizou-se um curso disponível na Coursera (COURSERA, 2021) "Neural Networks and Deep Learning" para introduzir as operações matemáticas, e os conceitos importantes para funcionamento e construção das redes neurais, como Loss Function, epochs, Logistic Regression, Activation Function, a quantidade de camadas utilizadas e entre outros conceitos.

A partir deste aprendizado inicial, atividades foram propostas para empregar os conceitos abordados e aplicá-los em duas frentes: uma sobre dados estruturados em planilhas e tabelas; e outra voltada a interpretação de imagens e símbolos.

Para construir uma rede neural com precisão é necessário aplicar uma quantidade de dados muito grande e bem estruturada para realizar o treino, assim foi utilizado uma base de dado pronta dentro da biblioteca Keras (KERAS, 2021) para a rede neural de classificação de imagens, e para de dados tabelados aplicou-se um dataset estruturado pelo Google APIs.

\section{Treinamento de Redes Neurais com Dados Estruturados}

Baseado em uma base de dados dos integrantes do Titanic (GOOGLE APIS, 2021), com o objetivo de predizer se um tripulante sobreviveu ou não, a partir dos dados presentes nesse dataset, como sexo, idade, de onde partiu, onde estava hospedado no navio, se estava acompanhado ou não. A base de dados para o treinamento e o teste também possuíam a informação se o tripulante havia ou não sobrevivido, dessa forma, foi possível verificar se a partir do treino a rede neural possuía uma boa predição ou não.

Buscando um melhor treinamento da rede neural, variou-se a quantidade nos batches e de epochs observando a precisão obtida para cada situação, assim obtivesse a maior precisão de $73,86 \%$ com 10 epochs sendo realizadas e cada batch contendo 32 dados de tripulantes.

\section{Treinamento de Redes Neurais com Imagens}

Apoiado pela biblioteca Keras, que apresenta diversos datasets que já acompanham suas funcionalidades, foram aplicados os conhecimentos de Machine Learning para imagens com o intuito de classificar uma base de dados de peças de roupas em suas respectivas classificações. O dataset utilizado foi o fashion_mist, contendo 10 classes de peças, por exemplo botas, tênis, camisetas, casacos, saias, calças, chinelos e dentre outras.

Para a construção desta rede neural foi necessário a utilização de 3 camadas, sendo a primeira responsável por achatar as imagens, pois estas contêm três dimensões o que poderia dificultar o processo de aprendizado da rede e diminuir a sua precisão. A segunda camada é responsável pela classificação das imagens de treino, aplicou-se a função de ativação $\operatorname{Re} L u$, pois esta apresentava maior precisão, comparada a Leaky $\operatorname{Re} L u$. A última camada da rede neural é a Softmax, responsável por apresentar o resultado obtido pelo treino realizado, por isso esta camada apresenta 10 nós, devido às dez classificações de peças de roupas que se deseja obter. Ao final do treino e realizou-se testes com uma base de dados também já pronta pelo Keras, obtivemos uma precisão de $87,66 \%$ na classificação das imagens. 


\subsection{Projeto: Estruturação de uma Machine Learning para Reconhecimento de Placas de Trânsito}

Partindo do conhecimento já desenvolvido anteriormente sobre Machine Learning, concentrou-se em aprofundar a construção das redes neurais a partir de um projeto de maior complexidade, visando uma implementação mais próxima do cotidiano. Dessa forma, elaborou-se um projeto de construção de uma rede neural para identificação de placas de trânsito pensando no futuro de carros autônomos e inteligentes, os quais conseguem capturar imagens ao seu redor e conseguir identificar as placas e interpretá-las. O projeto visou a interpretação dessas imagens.

Foi utilizado uma base de dados pronta com imagens de placas de trânsito na Bélgica (GEOAUTOMATION, 2021) para o treinamento e para o teste da rede neural, apresentando 62 categorias de placas como limites de velocidade, obras na via, faixa de pedestre, lombada, rotatória e entre tantas outras, sendo que cada categoria apresenta uma pasta com diversas imagens distintas, portanto, inicialmente juntando todas as imagens de cada pasta em uma única lista para facilitar o procedimento.

O estudo trouxe desafios: um deles foi sobre a uniformização das imagens contidas na base de dados para cada imagem padronizada com as dimensões de $32 \times 32$ pixels. Outro desafio encontrado durante o processo foi o tamanho do dataset disponível, apesar de apresentar imagens para cada categoria, algumas apresentavam poucas imagens para o treinamento, assim se desenvolveu e aplicou o conceito de data augmentation. Este processo é utilizado quando desejasse aumentar a base de dados a partir das imagens já contidas, utilizando recursos de variação do contraste das fotos, aplicando zoom, girando e entre outros recursos.

Para o projeto algumas funcionalidades não se aplicariam, como por exemplo a variação de cor e a rotação dificultariam o processo de aprendizado da rede neural, uma vez que o programa pode interpretar estas novas imagens geradas como uma outra categoria a qual esta não pertence. Em seguida, foi desenvolvido a rede neural para o projeto utilizando 4 camadas, sendo a primeira responsável pela ampliação da base de dados com o data augmentation, a segunda, assim como no projeto 5.2, para uniformizar os tamanhos das imagens. As duas últimas camadas são responsáveis por identificar as imagens em 62 categorias e devolver o resultado da classificação. Variando a quantidade de dados por batches e a quantidade de epochs necessárias para realizar o treinamento, foi alcançado a maior precisão de $85,3 \%$ para as imagens de treinamento com 20 epochs sendo realizadas e 32 dados por batch, assim como a melhor função de ativação foi a ReLu entre esta e a Leaky ReLu. Como resultado desta atividade, segundo o planejamento indicado no item 3 (Figura 4.5).

Figura 4.5 - Resultados obtidos da Meta 2.

\begin{tabular}{|l|l|l|l|l|}
\hline Meta Pedagógica: Iniciaço em IA e Inteligência Artificial e Machine Learning \\
\hline 1. Lembrar & 2. Entender & 3. Aplicar & 4. Avaliar & 5. Criar \\
\hline Iniciado & Iniciado & Iniciado & Iniciado \\
\hline Habilidades Adicionais (Softskills) & Visão de inovação \\
\hline Análise de Plataformas Digitais & $\begin{array}{l}\text { O estudante está com uma boa visão de que processos } \\
\text { tradicionais podem ser renovados e reinventados usando } \\
\text { dados e algoritmos. }\end{array}$ \\
\hline $\begin{array}{l}\text { As plataformas digitais constituem a base de novos negócios } \\
\text { baseados em jornadas de usuários e inteligência digital. }\end{array}$ \\
\hline
\end{tabular}

Segundo o estudante, o seguinte destaque: "Concluindo a última etapa do estudo, foi possível praticar a aplicação da Machine Learning em diversas áreas de aplicação, principalmente para modelos que apresentam grande enredamento dos fatores que 
influenciam no resultado desejado, e ao final, o aluno demonstrou conhecimento para aplicar os aprendizados de programação e IA em um projeto mais complexo e avaliar quais fatores podem ser alterados com o intuito de alcançar uma rede neural com maior precisão e efetividade."

\section{RESULTADOS OBTIDOS}

A metodologia proposta, o plano executado de aprendizado foi satisfatório. Em cada uma das etapas de aprendizagem, foi apresentada segundo a taxonomia de Bloom os avanços obtidos e com destaques também habilidades adicionais desenvolvidas no processo. Assim tivemos avanços segundo as atividades planejadas, ou seja: Na lógica de programação, o nível atingido foi a de entender e o de aplicar. Nesse ponto a abstração foi a base da metodologia; Na atividade de Implementação algorítmica, os níveis atendidos foram entender, aplicar, avaliar e criar. As técnicas de avaliar a estrutura e comportamento de um algoritmo para detectar erros e promover ajustes foi aprimorada; $\mathrm{Na}$ atividade de Serviços Web atingiu os níveis de avaliar e criar, ainda em fase básica conforme avaliação; $\mathrm{Na}$ atividade de Iniciação em Inteligência Artificial e Aprendizado de Máquina alcançou níveis adequados conforme o planejado que foi a de iniciar o estudante no assunto. O nível atingido foi a de aplicar e criar.

Habilidades adicionais muito importantes para a vida profissional do estudante também tiveram resultados expressivos tais como autonomia para buscar conhecimento, resiliência para resolução de problemas, capacidade de implementar além do projeto solicitado. A adoção de técnicas de inteligência artificial para construir modelos de negócios digitais também acrescentou uma visão ao aluno que pode contribuir, não somente no aproveitamento das diversas disciplinas da grade curricular, como também nos desafios profissionais desde os ciclos de estágios, incluindo o pensamento da inovação usando técnicas digitais.

\section{CONSIDERAÇÕES FINAIS}

Esse conhecimento será útil para o futuro profissional da Engenharia Química, considerando o desafio das indústrias na assimilação da tecnologia digital e dos conceitos da indústria 4.0 para obter soluções diferenciadas que resultam em maior precisão e produtividade baseados em modelos matemáticos mais sofisticados com a adoção de técnicas de inteligência artificial. Por exemplo, estudos desenvolvidos aplicando redes neurais para solucionar problemas com heterogeneidades e computar equacionamentos de modelagem a distribuição mássica molecular de peptídeos na produção de produtos lácteos (SOUSA, 2003). Nestes estudos, maior precisão e predição do comportamento real nas reações e nos reatores químicos foram obtidos com o uso de Machine Learning, e incluindo dados empíricos no processamento.

\section{REFERÊNCIAS}

\section{Livros:}

ARAKAKI, R.; ARAKAKI, J.; ANGERAMI, P. M.; AOKI O.; SALLES D. S. Fundamentos de Programação C - Técnicas e Aplicações, $2^{\text {a }}$ Ed. Rio de Janeiro:LTC Livros Técnicos e Científicos Editora, 1990.

PRESSMAN, R.S.; MAXIM, B.R. Software Engineering: a practitioner 's approach. $8^{a}$ Ed. New York: AMGH Editora Ltda., 2016. 


\section{Artigos de periódicos:}

SOUSA JR., Ruy et al. Hybrid Model for an Enzymatic Reactor: Hydrolysis of Cheese Whey Proteins by Alcalase Immobilized in Agarose Gel Particles. Humana Press Inc., [S. I.], ano 2003, v. 105-108, p. 413-422, 2003.

\section{Trabalhos em eventos}

Hayashi, V; Almeida, F; Arakaki, R; Teixeira, J. C; Martins, D; Midorikawa, E; Cugnasca, P. S; Canovas, S. LabEAD: Laboratório Remoto para o Ensino de Engenharia. In: S WORKSHOPS DO CONGRESSO BRASILEIRO DE INFORMÁTICA NA EDUCAÇÃO (WCBIE), 9., 2020, Online. Anais [...]. Porto Alegre: Sociedade Brasileira de Computação, 2020. p. 187-194. DOI: https://doi.org/10.5753/cbie.wcbie.2020.187.

VALENTE, José Armando. Aprendizagem Ativa no Ensino Superior: a proposta da sala de aula invertida. Depto. de Multimeios, Nied e GGTE-Unicamp \& Ced-PucSP, 2013.

FUJII, Tiago Yukio et al. Desafios para Aplicação de MLOps na Previsão do Consumo Energético. In: Conferência de Aplicação Industrial de Eletricidade, Conservação, Confiabilidade e Controle/Automação (INDUSCON). São Paulo, n. 14, 2021. No prelo.

\section{Internet:}

AMINI, Alexander. MIT 6.S191: Introduction to Deep Learning. Disponível em: https://www.youtube.com/watch?v=njKP3FqW3Sk. Acesso em: 15 mai. 2021.

COURSERA. Redes Neurais e Aprendizagem Profunda. Disponível em: https://pt.coursera.org/learn/neural-networks-deep-learning. Acesso em: 20 março 2021.

DEPARTAMENTO DE ENGENHARIA QUÍMICA DA UFSCAR. Curso de Graduação em Engenharia Química: Projeto Pedagógico. Disponível em:

http://www.prograd.ufscar.br/cursos/cursos-oferecidos-1/engenharia-quimica/engenhariaquimica-projeto-pedagogico.pdf. Acesso em: 5 maio 2021.

\section{DEPARTAMENTO DE ENGENHARIA QUÍMICA DA USP-LORENA. Escola de}

Engenharia de Lorena: Grade Curricular. Disponível em:

https://uspdigital.usp.br/jupiterweb/listarGradeCurricular?codcg=88\&codcur=88052\&codha b=1\&tipo=N. Acesso em: 5 maio 2021.

FGV - DISCIPLINA PIONEIRA CAPACITA ALUNOS EM PROGRAMAÇÃO PARA PRÁTICA JURÍDICA E EMPREENDEDORISMO

https://portal.fgv.br/noticias/disciplina-pioneira-capacita-alunos-programacao-praticajuridica-e-empreendedorismo. Acesso em: 05 de maio de 2021.

JUPITERWEB. Ementa da disciplina de Laboratório Digital I. Disponível em: https://uspdigital.usp.br/jupiterweb/obterDisciplina?nomdis=\&sgldis=PCS3635. Acesso em: 26 abril 2021.

KERAS SIG. Keras: Simple. Flexible. Powerful. Disponível em: https://keras.io/. Acesso em: 15 março 2021.

MONGODB, INC. MongoDB. Disponível em: https://www.mongodb.com/3. Acesso em: 15 março 2021. 
PYTHON SOFTWARE FOUNDATION. Flask: web development, one drop at a time. Disponível em: https://flask.palletsprojects.com/en/2.0.x/. Acesso em: 15 março 2021.

RIBEIRO, Jorge; LIMA, Rui; ECKHARDT, Tiago; PAIVA, Sara. Robotic Process Automation and Artificial Intelligence in Industry 4.0 - A Literature Review. Science Direct, 2020. Disponível em:

https://www.sciencedirect.com/science/article/pii/S1877050921001393b. Acesso em: 15 março 2021.

ZHUANG, Yueting; CAI, Ming; LI, Xuelong; LUO, Xiangang; YANG, Qiang; WU, Fei. The Next Breakthroughs of Artificial Intelligence: The Interdisciplinary Nature of Al. Science Direct, 2020. Disponível em:

https://www.sciencedirect.com/science/article/pii/S209580992030028X. Acesso em: 15 março 2021.

\section{Dados e softwares abertos:}

GEOAUTOMATION. BelgiumTS Dataset. Disponível em:

https://btsd.ethz.ch/shareddata/. Acesso em: 20 dezembro 2021.

GOOGLE APIS. Titanic Datasets. Disponível em: https://storage.googleapis.com/tfdatasets/titanic/train.csv. Acesso em: 15 março 2021.

\section{Systematic training of a Chemical Engineering Student to get into Computation Techniques}

Abstract: This paper describes results obtained from a student training in computer programming to improve algorithm design, implementation methods, error-correcting cycle, Artificial Intelligence concepts, Machine Learning configuration, and training. A learning plan, using Bloom Taxonomy, has been applied to get a consistent way to collect data about student challenges and improvements. The main activities brought scientific results and additional capability to student act in terms of autonomy, resilience to face errors and difficulties, and more fluency in IT systems design and implementation.

Keywords: Remote education, Education evaluation, Data Science, Programming Methods, Machine Learning, Data Processing Tools, Softskills. 